\title{
Research Article \\ On the Controllability of a Differential Equation with Delayed and Advanced Arguments
}

\author{
Raúl Manzanilla, Luis Gerardo Mármol, and Carmen J. Vanegas \\ Departamento de Matemáticas Puras y Aplicadas, Universdad Simón Bolívar, \\ Caracas 1080-A, Venezuela \\ Correspondence should be addressed to Luis Gerardo Mármol, lgmarmol@usb.ve \\ Received 4 January 2010; Revised 26 March 2010; Accepted 20 April 2010 \\ Academic Editor: Roman Šimon Hilscher \\ Copyright (C) 2010 Raúl Manzanilla et al. This is an open access article distributed under the \\ Creative Commons Attribution License, which permits unrestricted use, distribution, and \\ reproduction in any medium, provided the original work is properly cited.
}

A semigroup theory for a differential equation with delayed and advanced arguments is developed, with a detailed description of the infinitesimal generator. This in turn allows to study the exact controllability of the equation, by rewriting it as a classical Cauchy problem.

\section{Introduction}

In this paper, we will study the exact controllability of a functional differential equation with both delayed and advanced arguments. Such equations are often referred to in the literature as mixed-type functional differential equations (MTFDE) or forward-backward equations. The study of this type of equations is less developed compared with other classes of functional equations. Interest in MTDFEs is motivated by problems in optimal control [1] and applications, for example, in economic dynamics [2] and travelling waves in a spatial lattice [3]. See also [4]. In all these references, the reader can find interesting examples and applications.

In order to achieve our goal, first, we rewrite the equation as a classical Cauchy problem in a certain Banach space. Then we introduce the associated semigroup and its infinitesimal generator and prove some important properties of these operators (including some spectral properties). This will allow us to characterize the exact controllability, by applying a result of Bárcenas and Diestel (see [5]). 


\section{Preliminary Results}

In [6], the following differential-difference equation is considered

$$
x^{\prime}(t)=x(t-1)+x(t+1),
$$

where $t \geq 0$ and $x:[-1,+\infty) \rightarrow \mathbb{C}^{n}$ is differentiable in $[0,+\infty)$.

Equation (2.1) may be written as

$$
x(t+1)=x^{\prime}(t)-x(t-1)
$$

or equivalently

$$
x(t)=x^{\prime}(t-1)-x(t-2) .
$$

From this we have that in order to find the solution $x(t)$ on the interval $[m, m+1]$, it is necessary to know its value on the interval $[m-2, m]$, with $m$ being a positive integer. In particular, to determine the solution on the interval $[1,2]$, it is necessary to know it on the interval $[-1,1]$.

Accordingly, $x(t)$ is defined for $t \in[-1,1]$ as

$$
x(t)=\varphi(t)= \begin{cases}\varphi_{1}(t), & t \in[-1,0], \\ \varphi_{2}(t), & t \in[0,1],\end{cases}
$$

where the function $\varphi$ belongs to the space $C^{\infty}\left([-1,1], \mathbb{C}^{n}\right)$. The solution of the initial value problem $(2.1),(2.4)$ is constructed via an iterative process using the step derivation method.

It is

$$
x(t)=\sum_{i=0}^{m} c_{1_{i}} \varphi^{(i)}(t-m)+\sum_{i=0}^{m} c_{2_{i}} \varphi^{(i)}(t-m-1),
$$

where $c_{1_{i}}$ and $c_{2_{i}}$ are constants not all necessarily different from zero.

This solution may be extended to the left by rewriting (2.1) as

$$
x(t-1)=x^{\prime}(t)-x(t+1)
$$

which allows to yield an expression for $x(t)$ analogous to (2.5).

In order to assure the existence, differentiability, and uniqueness of the solution $x(t)$, it is demanded that $x(t)$ must satisfy the relationship

$$
\varphi^{(n)}(0)=\varphi^{(n-1)}(-1)+\varphi^{(n-1)}(1)
$$

for $n=1,2,3, \ldots$.

Further, if a differentiable solution $x(t)$ exists, then it belongs to the space $C^{\infty}([-1$, $\left.+\infty], \mathbb{C}^{n}\right)$ (see [6, Theorems 3.1 and 3.2]). 

$\left.\mathbb{C}^{n}\right)$ :

Let $M$ be the following nonempty, closed subspace of the topological space $C^{\infty}([-1,1]$,

$$
M=\left\{\varphi \in C^{\infty}\left([-1,1], \mathbb{C}^{n}\right): \varphi^{(n)}(0)=\varphi^{(n-1)}(-1)+\varphi^{(n-1)}(1), n=1,2,3, \ldots\right\}
$$

The space $C^{\infty}\left([-1,1], \mathbb{C}^{n}\right)$ is endowed with the topology induced by the following countable system of seminorms:

$$
P_{k}(f)=\max _{t \in[-1,1]}\left\|f^{(k)}(x)\right\|_{\mathbb{C}^{n},} \quad \text { for } k=0,1,2, \ldots
$$

The convergence in this topology means the uniform convergence of the function and each of its derivatives of any order. We denote

$$
\|f\|_{k}=\sum_{i=0}^{k} P_{i}(f), \quad \text { for each } k=0,1,2, \ldots
$$

A sequence $\left\{f_{n}\right\}_{n=1}^{\infty}$ converges to $f$ if and only if $\left\|f_{n}-f\right\|_{k}$ tends to 0 , as $n$ tends to infinity, for each $k$.

For each $t \geq 0$, the operator $T(t)$ is defined on the solutions $x(t)$ of (2.1) as follows:

$$
T(t) x(\theta)=x(t+\theta), \quad \theta \in[-1,1] .
$$

The following result originally appears on [7].

Theorem 2.1. The family $\{T(t)\}_{t \geq 0}$ defines a strongly continuous semigroup on $L(M)$.

Proof. That $T(0)=I$ and $T(t+s)=T(t) T(s)$ for each $t, s \geq 0$ are straightforward from the definition of $T(t)$. Since $\left.x(\theta)\right|_{\theta \in[-1,1]}=\varphi(\theta) \in M$, the domain of $T(t)$ is $M$. On the other hand, the function

$$
y_{t}(\theta):=T(t) x(\theta)=x(t+\theta)
$$

belongs to $M$ for each $t \geq 0$, because if $x(t) \in C^{\infty}\left([-1,1], \mathbb{C}^{n}\right)$, then $y_{t}(\theta) \in C^{\infty}\left([-1,1], \mathbb{C}^{n}\right)$ for each fixed $t \geq 0$. Additionally, for each $t \geq 0, y_{t}^{(n)}(0)=y_{t}^{(n-1)}(-1)+y_{t}^{(n-1)}(1)$, for $n=1,2,3, \ldots$, or equivalently

$$
x^{(n)}(t)=x^{(n-1)}(t-1)+x^{(n-1)}(t+1), \quad n=1,2, \ldots
$$

Hence $y_{t}(\theta) \in M$.

In order to prove that $T(\tau)$ is continuous for each fixed $\tau \geq 0$, we will prove that there exists $n_{k}$ for each $k$ and some constant $c \geq 0$ such that

$$
\|T(\tau) x(\theta)\|_{k} \leq c \sum_{i=0}^{n_{k}}\|x(\theta)\|_{i}
$$


In view that

$$
\begin{aligned}
P_{k}(T(\tau) x(\theta)) & =\max _{\theta \in[-1,1]}\left|x^{(k)}(\tau+\theta)\right| \leq \max _{t \in[[\tau]]-1,[\tau]+2]}\left|x^{(k)}(t)\right| \\
& \leq \max _{t \in[\tau]-1,[\tau]]}\left|x^{(k)}(t)\right|+\max _{t \in[[\tau]],[\tau]+1]}\left|x^{(k)}(t)\right|+\max _{t \in[[\tau]]+1,[\tau]+2]}\left|x^{(k)}(t)\right|
\end{aligned}
$$

and using formula (2.5) with $m=[\tau]$, one obtains

$$
\begin{aligned}
\max _{\theta \in[-1,1]}\left|x^{(k)}(\tau+\theta)\right| \leq & \sum_{i=0}^{m-1} c_{1_{i}} \max _{t \in[m-1, m]}\left|\varphi^{(i+k)}(t-(m-1))\right|+\sum_{i=0}^{m-1} c_{2_{i}} \max _{t \in[m-1, m]}\left|\varphi^{(i+k)}(t-(m-1)-1)\right| \\
& +\sum_{i=0}^{m} c_{3_{i}} \max _{t \in[m, m+1]}\left|\varphi^{(i+k)}(t-m)\right|+\sum_{i=0}^{m} c_{4_{i}} \max _{t \in[m, m+1]}\left|\varphi^{(i+k)}(t-m-1)\right| \\
& +\sum_{i=0}^{m+1} c_{5_{i}} \max _{t \in[m+1, m+2]}\left|\varphi^{(i+k)}(t-(m+1))\right| \\
& +\sum_{i=0}^{m+1} c_{6_{i}} \max _{t \in[m+1, m+2]}\left|\varphi^{(i+k)}(t-(m+1)-1)\right| \\
\leq & \sum_{i=0}^{m-1}\left(c_{1_{i}}+c_{2_{i}}\right) \sum_{i=0}^{m-1} \max _{\theta \in[-1,1]}\left|\varphi^{(i+k)}(\theta)\right|+\sum_{i=0}^{m}\left(c_{3_{i}}+c_{4_{i}}\right) \sum_{i=0}^{m} \max _{\theta \in[-1,1]}\left|\varphi^{(i+k)}(\theta)\right| \\
& +\sum_{i=0}^{m+1}\left(c_{5_{i}}+c_{6_{i}}\right) \sum_{i=0}^{m+1} \max _{\theta \in[-1,1]}\left|\varphi^{(i+k)}(\theta)\right| \\
\leq & c_{1}\|\varphi\|_{m+k-1}+c_{2}\|\varphi\|_{m+k}+c_{3}\|\varphi\|_{m+k+1} \\
\leq & \left(c_{1}+c_{2}+c_{3}\right)\left(\|\varphi\|_{m+k-1}+\|\varphi\|_{m+k}+\|\varphi\|_{m+k+1}\right) \\
= & c\left(\|\varphi\|_{m+k-1}+\|\varphi\|_{m+k}+\|\varphi\|_{m+k+1}\right) .
\end{aligned}
$$

It only remains to prove that $T(t) x \rightarrow T\left(t_{0}\right) x$, as $t \rightarrow t_{o}$, for each $x \in M$.

In fact, let $t_{0} \geq 0$. We have that $\lim _{t \rightarrow t_{0}} T(t) x=T\left(t_{0}\right) x \Leftrightarrow \lim _{\tau \rightarrow 0} T\left(t_{0}+\tau\right) x=T\left(t_{0}\right) x \Leftrightarrow$ $\left\|T\left(t_{0}+\tau\right) x(\theta)-T\left(t_{0}\right) x(\theta)\right\|_{k} \rightarrow 0$ as $\tau \rightarrow 0$ for each $k=0,1, \ldots, \Leftrightarrow \max _{\theta \in[-1,1]} \mid x^{(k)}\left(t_{0}+\tau+\right.$ $\theta)-x^{(k)}\left(t_{0}+\theta\right) \mid \rightarrow 0$ as $\tau \rightarrow 0$ for each $k=0,1, \ldots$

Assuming that $0 \leq[\tau] \leq 1$ and taking into account the uniform convergence of $x^{(k)}(t)$ in the closed interval $[a, b]:=\left[t_{0}-2, t_{0}+2\right]\left(t, t^{\prime} \in[a, b]\right)$, it follows that

$$
\max _{\theta \in[-1,1]}\left|x^{(k)}\left(t_{0}+\tau+\theta\right)-x^{(k)}\left(t_{0}+\theta\right)\right| \leq \max _{\left|t-t^{\prime}\right| \leq[\tau]}\left|x^{(k)}(t)-x^{(k)}\left(t^{\prime}\right)\right| \longrightarrow 0
$$

as $\tau \rightarrow 0$. 
Some basic definitions and concepts on controllability are recalled below. Let $U$ and $X$ be Banach spaces. We consider the inhomogeneous differential linear system:

$$
\begin{gathered}
\dot{x}(t)=A x(t)+B u(t), \\
x(0)=x_{0} \in D(A),
\end{gathered}
$$

where $A: X \rightarrow X$ is the infinitesimal generator operator of a strongly continuous semigroup $\left(S_{t}\right)_{t \geq 0} ; B: U \rightarrow U$ is a bounded linear operator and $u:[0,+\infty) \rightarrow U$ is a strongly measurable essentially bounded function.

Let $\Omega$ be a nonempty separable weakly compact convex subset of $U$.

We recall (see [8]) that $x_{0} \in X$ is controllable with respect to $x_{1}$ if there exist $\bar{t} \geq 0$ and a control $u \in L_{\infty}([0, \bar{t}] ; U)$ such that $x(\bar{t})=x_{1}$ in $(2.18)$.

The controllability map on $[0, \bar{t}]$ for some $\bar{t} \geq 0$ is the linear map

$$
B^{\bar{t}}: L_{\infty}([0, \bar{t}] ; U) \longrightarrow X
$$

defined by

$$
B^{\bar{t}} u=\int_{0}^{\bar{t}} S(\bar{t}-s) B u(s) \mathrm{d} s
$$

Now, one says that $(2.18)$ is exactly controllable on $[0, \bar{t}]$ if every point in $X$ can be reached from the origin at time $\bar{t}$, that is, if $\operatorname{ran}\left(B^{\bar{t}}\right)=X$, which is equivalent to

$$
\begin{aligned}
x_{1} & =\int_{0}^{\bar{t}} S(\bar{t}-s) B u(s) \mathrm{d} s, \\
0 & =-x_{1}+\int_{0}^{\bar{t}} S(\bar{t}-s) B u(s) \mathrm{d} s, \\
\text { or } \quad 0 & =S(\bar{t}) x_{0}+\int_{0}^{\bar{t}} S(\bar{t}-s) B u(s) \mathrm{d} s .
\end{aligned}
$$

In other words,

$$
\operatorname{ran}\left(B^{\bar{t}}\right)=X \Longleftrightarrow 0 \in\left\{S(\bar{t}) x_{0}+\int_{0}^{\bar{t}} S(\bar{t}-s) B u(s) \mathrm{d} s: u \text { is a control in } L_{\infty}([0, \bar{t}] ; U)\right\} .
$$

The set $\Omega_{\bar{t}}=\left\{u \in L_{\infty}([0, t] ; U): u \in \Omega\right.$ a.e. $\}$ is called the set of admissible controls of (2.18), while the set $A_{\bar{t}}\left(x_{0}\right)=\left\{S(\bar{t}) x_{0}+\int_{0}^{\bar{t}} S(\bar{t}-s) B u(s) \mathrm{d} s: u \in \Omega_{\bar{t}}\right\}$ is called the set of accessible points of (2.18). Therefore, the system (2.18) is controllable if $0 \in A_{\bar{t}}\left(x_{0}\right)$, for each $\bar{t}>0$.

We will make use of the following theorem, which will be applied to problem (2.1),(2.4). 
Theorem 2.2 (Bárcenas and Diestel [5]). Let $X$ and $U$ be Banach spaces. Let $B: U \rightarrow X$ be a bounded linear operator and $A: X \rightarrow X$ the infinitesimal generator of a $C_{0}$-semigroup $\left(S_{t}\right)_{t \geq 0}$ on $X$ whose dual semigroup is strongly continuous on $(0,+\infty)$. Suppose $\Omega$ is nonempty separable weakly compact convex subset of $U$ containing 0 . Then, for each $\bar{t}>0,0 \in A_{\bar{t}}\left(x_{0}\right)$ if and only if for each $x^{*} \in X^{*}$,

$$
x^{*}\left(S(\bar{t}) x_{0}\right)+\int_{0}^{\bar{t}} \max _{v \in \Omega} x^{*}(S(t) B v) \mathrm{d} t \geq 0
$$

The Bárcenas-Diestel Theorem is an important and recent achievement on exact controllability. Throughout the literature on optimal control in Banach Spaces, hypotheses like "separable and reflexive" are frequently encountered. Using techniques from Banach space theory and the theory of vector measures, the authors show how to remove the hypothesis of reflexivity (thus giving considerably greater generality to the resulting conclusions) and translate the question of accessibility of controls to a problem in semigroups of operators, namely, given a $c_{0}$-semigroup $(S(t))_{t \geq 0}$ of operators on a Banach space $X$, under what conditions is the dual semigroup strongly continuous on $(0, \infty)$ ?

It should be noted that, for each fixed $t \geq 0$ and each $x^{*} \in X^{*}$, a bounded linear functional $u^{*} \in U^{*}$ is defined by means of $u^{*}(v):=x^{*}(S(t) B v)$. The maximum in Theorem 2.2 exists as a consequence of a now classical result of James [9], stating that a weakly closed subset $C$ of a Banach space $Z$ is weakly compact if and only if each continuous linear functional on $Z$ attains a maximum on $C$.

On the other hand, the following result is proven in [10]: for each $x^{*} \in X^{*}$, the mapping of $[0, \tau]$ to $[0, \infty)$ that takes $t \in[0, \tau]$ to $\max _{v \in \Omega} x^{*}(S(t) B v)$ is continuous (see also [5]), and so the integral in Theorem 2.2 exists in the common Riemann sense.

\section{The Cauchy Problem}

We will formulate the problem (2.1),(2.4) in the form (2.18). One should observe that we are working on a topological space which is not a Banach space. Let us consider the space $\mathbb{C}^{n} \times M$, endowed with the product topology, where $M$ is defined in (2.8), and let $N$ be the closed subspace of all pairs $(r, f)$ in $\mathbb{C}^{n} \times M$ such that $f(0)=r$. On $N$ we define the following map:

$$
S(t)\left(\begin{array}{c}
\varphi(0) \\
\varphi(\cdot)
\end{array}\right)=\left(\begin{array}{c}
x(t) \\
x(t+\theta)
\end{array}\right)=\left(\begin{array}{c}
\sum_{i=0}^{m} c_{1_{i}} \varphi^{(i)}(t-m)+\sum_{i=0}^{m} c_{2_{i}} \varphi^{(i)}(t-m-1) \\
x(t+\theta)
\end{array}\right)
$$

for each $t \geq 0$ and $\theta \in[-1,1]$, where $x(t)$ is the solution (2.5) of the initial value problem $(2.1),(2.4)$.

Now, one has the following result.

Theorem 3.1. The operator $S$ satisfies

(i) $S(t) \in L(N)$ for all $t \geq 0$;

(ii) $S(t)$ is a $C_{0}$-semigroup in $N$. 
Proof. (i) The linearity of $S(t)$ follows from the linearity of its components. In order to see the continuity for each $t \geq 0$, let us bear in mind that we are working with the product topology. The continuity and convergence for this topology are coordinatewise. We say that $(f(0), f)$ is close or tends to $(g(0), g)$ if $\|f(0)-g(0)\|_{\mathbb{C}^{n}}$ is close to 0 and $\|f-g\|_{k}$ is close to 0 , for each $k$, $(k=0,1,2, \ldots)$, or, equivalently, if for each $k$

$$
\|f(0)-g(0)\|_{\mathbb{C}^{n}}+\|f-g\|_{k}
$$

is close to 0 , where $\|\cdot\|_{k}$ is defined as above. In the case of the linear map $S(t)$, the second coordinate is the continuous semigroup (2.11), and so, if $c_{1}=\max _{1 \leq i \leq m}\left|c_{1_{i}}\right|$ and $c_{2}=\max _{1 \leq i \leq m}\left|c_{2_{i}}\right|$, we have, for each $k$

$$
\begin{aligned}
\|x(t)\|_{\mathbb{C}^{n}}+\|x(t+\theta)\|_{k} & \leq \sum_{i=0}^{m} c_{1}\left\|\varphi^{(i)}(t-m)\right\|_{\mathbb{C}^{n}}+\sum_{i=0}^{m} c_{2}\left\|\varphi^{(i)}(t-m-1)\right\|_{\mathbb{C}^{n}}+c \sum_{i=0}^{n_{k}}\|\varphi(\cdot)\|_{i} \\
& \leq\left(c_{1}+c_{2}\right)\|\varphi(\cdot)\|_{m}+c \sum_{i=0}^{n_{k}}\|\varphi(\cdot)\|_{i} .
\end{aligned}
$$

If $(\varphi(0), \varphi(\cdot))$ is close to $(0,0)$, then, in particular, $\|\varphi(\cdot)\|_{k}$ is close to 0 , for each $k$, and thus, by the previous estimate, $S(t)\left(\begin{array}{c}\varphi(0) \\ \varphi(\cdot)\end{array}\right)$ is close to $(0,0)$. Being $S(t)$ linear, this is enough to prove that it is continuous.

(ii) Now we will check the semigroup properties. Obviously, $S(0)=I$. To prove $S(t+$ $s)=S(t) S(s)$, one defines the function $h(t)=x(t+s)$, where $x(\cdot)$ is the solution of $(2.1),(2.4)$. Therefore, $h(t)$ satisfies

$$
\begin{aligned}
& \dot{h}(t)=h(t-1)+h(t+1), \quad 0 \leq t, \\
& h(0)=x(s), \\
& h(\theta)=\varphi(s+\theta), \quad \theta \in[-1,1] .
\end{aligned}
$$

By the definition of $S$, one has

$$
\left(\begin{array}{c}
h(t) \\
h(t+\cdot)
\end{array}\right)=S(t)\left(\begin{array}{c}
x(s) \\
x(s+\cdot)
\end{array}\right)=S(t) S(s)\left(\begin{array}{c}
\varphi(s) \\
\varphi(\cdot)
\end{array}\right)
$$

On the other hand,

$$
S(t+s)\left(\begin{array}{c}
\varphi(0) \\
\varphi(\cdot)
\end{array}\right)=\left(\begin{array}{c}
x(t+s) \\
x(t+s+\cdot)
\end{array}\right)=\left(\begin{array}{c}
h(t) \\
h(t+\cdot)
\end{array}\right)=S(t) S(s)\left(\begin{array}{c}
\varphi(0) \\
\varphi(\cdot)
\end{array}\right) .
$$

According to Theorem 2.1, we have that $\lim _{t \rightarrow 0^{+}} x(t+s)=x(s)$; since $\varphi$ is continuous, then $\lim _{t \rightarrow 0^{+}} x(t)=\varphi(0)$. Consequently, $\lim _{t \rightarrow 0^{+}} S(t)=I$ and $S(t)$ is a $C_{0}$-semigroup in $N$. 
Lemma 3.2. Let $A$ be the infinitesimal generator associated to the semigroup $S(t)$. For $\alpha \in \mathbb{R}$ sufficiently large, the resolvent is given by

$$
(\alpha I-A)^{-1}\left(\begin{array}{c}
\varphi(0) \\
\varphi(\cdot)
\end{array}\right)=\left(\begin{array}{c}
g(0) \\
g(\cdot)
\end{array}\right)
$$

where

(i) $g(\theta)=e^{\alpha \theta} g(0)-\int_{0}^{\theta} e^{\alpha(\theta-s)} \varphi(s) \mathrm{d} s, \theta \in[-1,1]$;

(ii) $g(0)=(\Delta(\alpha))^{-1}\left[\varphi(0)+\int_{-1}^{0} e^{-\alpha(\theta+1)} \varphi(\theta) \mathrm{d} \theta-\int_{0}^{1} e^{-\alpha(\theta-1)} \varphi(\theta) \mathrm{d} \theta\right]$ with $\Delta(\lambda)=\left(\lambda-e^{\lambda}-\right.$ $\left.e^{-\lambda}\right) I$, for $\lambda \in C$.

Further, $g$ satisfies the following relation:

(iii) $\alpha g(0)=\varphi(0)+g(-1)+g(1)$.

Proof. (i) From [8, Lemma 2.1.11], for $\alpha \geq \omega_{0}$ ( $\omega_{0}$ is the growth bound of the semigroup), one has

$$
(\alpha I-A)^{-1}\left(\begin{array}{c}
\varphi(0) \\
\varphi(\cdot)
\end{array}\right)=\int_{0}^{+\infty} e^{-\alpha t} S(t)\left(\begin{array}{c}
\varphi(0) \\
\varphi(\cdot)
\end{array}\right) \mathrm{d} t=\int_{0}^{+\infty} e^{-\alpha t}\left(\begin{array}{c}
x(t) \\
x(t+\cdot)
\end{array}\right) \mathrm{d} t
$$

One defines for $\theta \in[-1,1]$

$$
g(\theta)=\int_{0}^{+\infty} e^{-\alpha t} x(t+\theta) \mathrm{d} t=\int_{\theta}^{+\infty} e^{-\alpha(s-\theta)} x(s) \mathrm{d} s .
$$

It is observed that $g(\theta)$ is a solution of the differential equation

$$
\frac{\mathrm{d} g}{\mathrm{~d} \theta}(\theta)=\alpha g(\theta)-x(\theta)
$$

The variation of constants formula for (3.10) shows that $g(\theta)$ is equal to (i).

(iii) Since, as it was defined above,

$$
g(\theta)=\int_{0}^{+\infty} e^{-\alpha t} x(t+\theta) \mathrm{d} t, \quad \theta \in[-1,1]
$$

one has after an integration by parts,

$$
\alpha g(0)=\alpha \int_{0}^{+\infty} e^{-\alpha t} x(t) \mathrm{d} t=\varphi(0)+\int_{0}^{+\infty} e^{-\alpha t}(x(t-1)+x(t+1)) \mathrm{d} t=\varphi(0)+g(-1)+g(1)
$$

which is (iii) 
(ii) On the other hand,

$$
\begin{aligned}
\alpha g(0) & =\varphi(0)+\int_{0}^{-1} e^{-\alpha t} x(t+1) \mathrm{d} t+\int_{-1}^{+\infty} e^{-\alpha t} x(t+1) \mathrm{d} t+\int_{0}^{1} e^{-\alpha t} x(t-1) \mathrm{d} t+\int_{1}^{+\infty} e^{-\alpha t} x(t-1) \mathrm{d} t \\
& =\varphi(0)+\int_{-1}^{+\infty} e^{-\alpha t} x(t+1) \mathrm{d} t+\int_{1}^{+\infty} e^{-\alpha t} x(t-1) \mathrm{d} t+\int_{0}^{1} e^{-\alpha t} \varphi(t-1) \mathrm{d} t-\int_{-1}^{0} e^{-\alpha t} \varphi(t+1) \mathrm{d} t \\
& =\varphi(0)+e^{\alpha} g(0)+e^{-\alpha} g(0)+\int_{0}^{1} e^{-\alpha t} \varphi(t-1) \mathrm{d} t-\int_{-1}^{0} e^{-\alpha t} \varphi(t+1) \mathrm{d} t .
\end{aligned}
$$

Therefore,

$$
\left[\left(\alpha-\left(e^{\alpha}+e^{-\alpha}\right)\right) I\right] g(0)=\varphi(0)+\int_{-1}^{0} e^{-\alpha(\theta+1)} \varphi(\theta) \mathrm{d} \theta-\int_{0}^{1} e^{-\alpha(\theta-1)} \varphi(\theta) \mathrm{d} \theta
$$

which is (ii) for $\alpha$ sufficiently large.

Theorem 3.3. Let $S(t)$ be the semigroup defined by (3.1). Then its infinitesimal generator is given by

$$
A\left(\begin{array}{c}
\varphi(0)=r \\
\varphi(\cdot)
\end{array}\right)=\left(\begin{array}{c}
\varphi(-1)+\varphi(1) \\
\frac{\mathrm{d} \varphi}{\mathrm{d} \theta}(\cdot)
\end{array}\right)
$$

where the domain of $A$ is

$$
D(A)=\left\{\left(\begin{array}{c}
r \\
\varphi(\cdot)
\end{array}\right) \in N: \varphi \text { is absolutely continuous, and } \frac{\mathrm{d} \varphi}{\mathrm{d} \theta} \in M\right\}
$$

Proof. Consider the operator $\widetilde{A}$ defined by

$$
\tilde{A}\left(\begin{array}{c}
r \\
\varphi(\cdot)
\end{array}\right)=\left(\begin{array}{c}
\varphi(-1)+\varphi(1) \\
\frac{\mathrm{d} \varphi}{\mathrm{d} \theta}(\cdot)
\end{array}\right)
$$

with

$$
D(\tilde{A})=\left\{\left(\begin{array}{c}
r \\
\varphi(\cdot)
\end{array}\right) \in N: \varphi \text { is absolutely continuous, and } \frac{\mathrm{d} \varphi}{\mathrm{d} \theta} \in M\right\} .
$$


We will prove that $A=\tilde{A}$. Suppose $\alpha$ is a sufficiently large number such that Lemma 3.2 is true. In this case, for $\left(\begin{array}{c}r \\ \varphi(\cdot)\end{array}\right) \in N$, one can write

$$
(\alpha I-\tilde{A})(\alpha I-A)^{-1}\left(\begin{array}{c}
r \\
\varphi(\cdot)
\end{array}\right)=(\alpha I-\tilde{A})\left(\begin{array}{c}
g(0) \\
g(\cdot)
\end{array}\right)=\left(\begin{array}{c}
\alpha g(0)-g(1)-g(-1) \\
\alpha g(\cdot)-\frac{\mathrm{d} g}{\mathrm{~d} \theta}(\cdot)
\end{array}\right)=\left(\begin{array}{c}
r \\
\varphi(\cdot)
\end{array}\right)
$$

by differentiating (i) from Lemma 3.2.

Now, let us see that

$$
(\alpha I-A)(\alpha I-\tilde{A})^{-1}\left(\begin{array}{c}
r \\
\varphi(\cdot)
\end{array}\right)=\left(\begin{array}{c}
r \\
\varphi(\cdot)
\end{array}\right)
$$

To do this, one takes $\left(\begin{array}{c}r \\ \varphi(\cdot)\end{array}\right) \in D(A)$ and defines

$$
\left(\begin{array}{c}
r_{1} \\
\varphi_{1}(\cdot)
\end{array}\right):=(\alpha I-A)^{-1}(\alpha I-\tilde{A})\left(\begin{array}{c}
r \\
\varphi(\cdot)
\end{array}\right)
$$

We will show that $(\alpha I-\tilde{A})$ is injective.

Suppose that there exists $\left(\begin{array}{c}r_{0} \\ \varphi_{0}(\cdot)\end{array}\right) \in D(A)$ such that

$$
\left(\begin{array}{l}
0 \\
0
\end{array}\right)=(\alpha I-\tilde{A})\left(\begin{array}{c}
r_{0} \\
\varphi_{0}(\cdot)
\end{array}\right)=(\alpha I-\tilde{A})\left(\begin{array}{c}
\varphi_{0}(0) \\
\varphi_{0}(\cdot)
\end{array}\right)=\left(\begin{array}{c}
\alpha \varphi_{0}(0)-\varphi_{0}(-1)-\varphi_{0}(1) \\
\alpha \varphi_{0}(\cdot)-\frac{\mathrm{d} \varphi_{0}}{\mathrm{~d} \theta}(\cdot)
\end{array}\right)
$$

which implies

$$
\begin{gathered}
\alpha \varphi_{0}(0)-\varphi_{0}(-1)-\varphi_{0}(1)=\alpha \varphi_{0}(0)-\varphi_{0}(0) e^{\alpha}-\varphi_{0}(0) e^{-\alpha}=\left(\alpha-e^{\alpha}-e^{-\alpha}\right) \varphi_{0}(0)=0, \\
\varphi_{0}(\theta)=\varphi_{0}(0) e^{\alpha \theta} .
\end{gathered}
$$

As $\alpha-e^{\alpha}-e^{-\alpha}$ is different from zero, then $\varphi_{0}(0)=r_{0}=0$ and so $\varphi_{0}(\cdot)=\varphi_{0}(0) e^{\alpha(\cdot)}=0$; thus $(\alpha I-\tilde{A})$ is injective. Consequently,

$$
(\alpha I-\tilde{A})\left(\begin{array}{c}
r_{1} \\
\varphi_{1}(\cdot)
\end{array}\right)=(\alpha I-\tilde{A})(\alpha I-A)^{-1}\left[(\alpha I-\tilde{A})\left(\begin{array}{c}
r \\
\varphi(\cdot)
\end{array}\right)\right]=(\alpha I-\tilde{A})\left(\begin{array}{c}
r \\
\varphi(\cdot)
\end{array}\right)
$$

which implies that

$$
\left(\begin{array}{c}
r_{1} \\
\varphi_{1}(\cdot)
\end{array}\right)=\left(\begin{array}{c}
r \\
\varphi(\cdot)
\end{array}\right)
$$


In other words, for every $\left(\begin{array}{c}r \\ \varphi(\cdot)\end{array}\right) \in D(A)$, one has

$$
(\alpha I-A)^{-1}(\alpha I-\tilde{A})\left(\begin{array}{c}
r \\
\varphi(\cdot)
\end{array}\right)=\left(\begin{array}{c}
r \\
\varphi(\cdot)
\end{array}\right)
$$

that it is equivalent to

$$
(\alpha I-\tilde{A})\left(\begin{array}{c}
r \\
\varphi(\cdot)
\end{array}\right)=(\alpha I-A)\left(\begin{array}{c}
r \\
\varphi(\cdot)
\end{array}\right)
$$

Then $\widetilde{A}=A$, as we wanted to see.

For each $m \in \mathbb{N}$, let $M_{m}$ be the space $M$ defined in (2.8) provided with the topology of $C^{m}\left([-1,1], \mathbb{C}^{n}\right)$, as a closed subset of $C^{m}\left([-1,1], \mathbb{C}^{n}\right) . M_{m}$ is a Banach space. Therefore, $\mathbb{C}^{n} \times M_{m}$, for all $m \in \mathbb{N}$, is also a Banach space with the norm

$$
\left\|\left(\begin{array}{l}
r \\
f
\end{array}\right)\right\|_{\mathbb{C}^{n} \times M_{m}}=\|r\|_{\mathbb{C}^{n}}+\|f\|_{\mathbb{C}^{m}\left([-1,1], \mathbb{C}^{n}\right)} .
$$

Now, let $N_{m}$ be the closed subspace of $\mathbb{C}^{n} \times M_{m}$ of all pairs $(r, f)$ such that $r=f(0)$.

The semigroup $S^{m}(t): N_{m} \rightarrow N_{m}$, defined in the same form as $S(t)$, is now a semigroup defined on a Banach space for all $m \in \mathbb{N}$.

Lemma 3.4. If $A^{m}$ denote the infinitesimal generator of $S^{m}(t)$, then $A^{m}=A$ for all $m \in \mathbb{N}$, where $A$ is the infinitesimal generator of $S(t)$.

Proof.

$$
\begin{aligned}
\lim _{t \rightarrow 0} \frac{1}{t} & \left\|S^{m}(t)\left(\begin{array}{c}
\varphi(0) \\
\varphi(\cdot)
\end{array}\right)-\left(\begin{array}{c}
\varphi(0) \\
\varphi(\cdot)
\end{array}\right)-t A\left(\begin{array}{c}
\varphi(0) \\
\varphi(\cdot)
\end{array}\right)\right\|_{\mathbb{C}^{n} \times M_{m}} \\
& =\lim _{t \rightarrow 0} \frac{1}{t}\left\|\left(\begin{array}{c}
x(t) \\
x(t+\theta)
\end{array}\right)-\left(\begin{array}{c}
\varphi(0) \\
\varphi(\cdot)
\end{array}\right)-\left(\begin{array}{c}
t(\varphi(-1)+\varphi(1)) \\
t \frac{\mathrm{d} \varphi}{\mathrm{d} \theta}(\cdot)
\end{array}\right)\right\|_{\mathbb{C}^{n} \times M_{m}} \\
& =\lim _{t \rightarrow 0}\left[\frac{1}{t}\|x(t)-\varphi(0)-t(\varphi(-1)+\varphi(1))\|_{\mathbb{C}^{n}}+\frac{1}{t}\left\|x(t+\theta)-\varphi(\cdot)-t \frac{\mathrm{d} \varphi}{\mathrm{d} \theta}(\cdot)\right\|_{M_{m}}\right] \\
& \leq \lim _{t \rightarrow 0}\left[\frac{1}{t}\|x(t)-\varphi(0)-t(\varphi(-1)+\varphi(1))\|_{\mathbb{C}^{n}}+\frac{1}{t} \sum_{k=0}^{m} P_{k}\left(x(t+\theta)-\varphi(\cdot)-t \frac{\mathrm{d} \varphi}{\mathrm{d} \theta}(\cdot)\right)\right]=0 .
\end{aligned}
$$

Theorem 3.5. Let $A$ be the infinitesimal generator of $S(t)$.

The spectrum of $A$ is discrete and it is defined by

$$
\sigma(A)=\{\lambda \in \mathbb{C}: \operatorname{det}(\Delta \lambda)=0\},
$$


where $\Delta \lambda=\left(\lambda-e^{\lambda}-e^{-\lambda}\right) I$ for all $\lambda \in \mathbb{C}$, and the multiplicity of each eigenvalue is finite. For every $\delta \in \mathbb{R}$, there exist only a finite number of eigenvalues in $C_{\delta}^{+}=\{s \in \mathbb{C}: \operatorname{Re}(s)>\delta\}$. If $\lambda \in \sigma(A)$, then $\left(\begin{array}{c}r \\ e^{\lambda} \cdot r\end{array}\right)$, where $r \neq 0$ satisfies $\Delta \lambda r=0$, is an eigenvector of $A$ with eigenvalue $\lambda$. On the other hand, if $\Phi$ is an eigenvector of $A$ with eigenvalue $\lambda$, then $\Phi=\left(\begin{array}{c}r \\ e^{\lambda} r\end{array}\right)$ with $\Delta \lambda r=0$.

Proof. According to the previous Lemma 3.2, for $\alpha \in \mathbb{R}$ sufficiently large, one has

$$
(\alpha I-A)^{-1}\left(\begin{array}{c}
r \\
\varphi(\cdot)
\end{array}\right)=\left(\begin{array}{c}
g(0) \\
g(\cdot)
\end{array}\right)
$$

where $g(\theta)$ and $g(0)$ are as (i) and (ii), respectively in Lemma 3.2. Denote by $Q_{\lambda}$ the extension of (3.31) to $\mathbb{C}$, that is,

$$
Q_{\lambda}\left(\begin{array}{c}
r \\
\varphi(\cdot)
\end{array}\right):=\left(\begin{array}{c}
g(0) \\
g(\cdot)
\end{array}\right)
$$

A simple calculation shows that if $\lambda \in \mathbb{C}$ satisfies $\operatorname{det}(\Delta \lambda) \neq 0$, then $Q_{\lambda}$ is a bonded linear operator from $N$ to $N$. Furthermore, for these $\lambda$ we have $(\lambda I-A) Q_{\lambda}=I$ and $(\lambda I-A)$ is injective. Therefore, $Q_{\lambda}=(\lambda I-A)^{-1}=Q(A)$, the resolvent operator of $A$.

Since $\{\lambda \in \mathbb{C}: \operatorname{det}(\Delta \lambda) \neq 0\} \subseteq \rho(A)$, then $\sigma(A) \subset\{\lambda \in \mathbb{C}: \operatorname{det}(\Delta \lambda)=0\}$.

On the other hand, if $\operatorname{det}(\Delta \mathcal{\lambda})=0$, then there exists a $\xi \in \mathbb{C}^{n}$ such that $\left(\lambda I-e^{-\lambda}\right.$ $\left.-e^{\lambda}\right)(\xi)=0$.

The following element of $N, z_{0}=\left(\begin{array}{c}\xi \\ e^{l \cdot \xi}\end{array}\right)$ belongs to $D(A)$ and

$$
(\lambda I-A) z_{0}=\left(\begin{array}{c}
\lambda \xi-e^{-\lambda} \xi-e^{\lambda} \xi \\
\lambda e^{\lambda \theta} \xi-\frac{\mathrm{d}}{\mathrm{d} \theta} e^{\lambda \theta} \xi
\end{array}\right)=\left(\begin{array}{l}
0 \\
0
\end{array}\right) .
$$

Then $\{\lambda \in \mathbb{C}: \operatorname{det}(\Delta \lambda)=0\} \subset \sigma(A)$.

Let $\lambda$ be an element in $C_{\delta}^{+}$with $e^{\delta}+e^{-\delta}<|\lambda|$.

For this $\lambda$, one has $\left|e^{\lambda}+e^{-\lambda}\right| \leq e^{\delta}+e^{-\delta}<|\lambda|$, and from [8, Corollary A.4.10], one concludes that $\lambda I-e^{\lambda}-e^{-\lambda}$ is invertible in $N_{m}$, where $N_{m}$ is defined as before. Thus $\operatorname{det}(\Delta \lambda) \neq 0$ and $\lambda \in \rho(A)$. Since $\operatorname{det}\left(\lambda I-e^{\lambda}-e^{-\lambda}\right)$ is an entire function, it has finitely many zeros in the compact set $\overline{C_{\delta}^{+}} \cap\left\{\lambda \in \mathbb{C}:|\lambda| \leq e^{\delta}+e^{-\delta}\right\}$ (see in [8, Theorem A.1.4.6b]) and we have showed that in the rest of $C_{\delta}^{+}$there are none. Therefore, there are only a finite number of eigenvalues in $C_{\delta}^{+}$.

Let $\Phi=\left(\begin{array}{c}r \\ \varphi(\cdot)\end{array}\right)$ be an eigenvector of $A$ with eigenvalue $\lambda$. From the definition of $A$, one obtains $(\mathrm{d} \varphi / \mathrm{d} \theta)(\theta)=\lambda \varphi(\theta)$ for $\theta \in[-1,1]$ which gives $\varphi(\theta)=e^{\lambda \theta} \varphi(0)$.

Since $\Phi \in D(A)$, one has $\varphi(0)=r$. Using the first equation of the definition of $A$, $e^{\lambda}+e^{-\lambda} r=\lambda r$ which shows that $\Delta \lambda r=0$. The other implication is obvious.

Finally, we will show that the multiplicity of each eigenvalue is finite. From Lemma 3.2, one has

$$
(\alpha I-A)^{-1}\left(\begin{array}{c}
\varphi(0) \\
\varphi(\cdot)
\end{array}\right)=\left(\begin{array}{c}
g(0) \\
e^{\alpha} g(0)
\end{array}\right)+\left(\begin{array}{c}
0 \\
-\int_{0} e^{\alpha(\cdot-s)} \varphi(s) \mathrm{d} s
\end{array}\right)
$$


where $g(0)$ is given in (ii) of Lemma 3.2. We deduce from this expression that the resolvent operator, as an operator from $N_{m}$ to $N_{m}$, is the sum of an operator with finite range and an integral operator. The first operator is compact (see [8, Lemma A.3.22a]), and so is the second one, as we will see. Therefore, $(\alpha I-A)^{-1}$ is compact as an operator of $L\left(N_{m}\right)$, for all $m \in \mathbb{N}$. From [8, Theorem A.4.18 and Lemma A.4.19], one obtains that the eigenvalue's multiplicity is finite for $(\alpha I-A)$ and $A$.

In order to prove the compactness of the second operator, let us observe that in [11, Example 1, page 277], it is seen that the operator

$$
T \varphi(a)=\int_{0}^{a} e^{\alpha(a-s)} \varphi(s) \mathrm{d} s
$$

is compact in $C[0, a]$. Let us suppose that $a \leq 1$ and let $B$ be the closed unit ball in $C^{m}[-1,1]$; then $B \cap M$ is the unit ball of $M_{m}$ and it is valid that $\overline{T(B \cap M)} \subseteq \overline{T(B)}$ in the topology of $C^{m}[-1,1]$.

Therefore, it is enough to prove that $\overline{T(\mathcal{B})}$ is compact in $C^{m}[-1,1]$. Let $\mathbb{B}^{\prime}$ be the closed unit ball of $C[0, a]$; then $\mathbb{B} \subseteq \mathbb{B}^{\prime}$ which implies $T(\mathbb{B}) \subseteq T\left(\mathbb{B}^{\prime}\right)$. If $\mathcal{W}$ is the closure of $T(\mathbb{B})$ in $C[0, a]$, then, as $T(\mathbb{B}) \subseteq T\left(\mathbb{B}^{\prime}\right), \mathcal{W}$ is compact in $C[0, a]$.

Now, let $\left\{V_{\alpha}\right\}_{\alpha \in J}$ be a covering consisting of open balls in $C^{m}[-1,1]$ of $\mathcal{W}$. Each $V_{\alpha}$ is contained in an open ball $\boldsymbol{B}_{\alpha}$ of $C[0, a]$. Therefore, $\left\{\boldsymbol{B}_{\alpha}\right\}_{\alpha \in J}$ is an open covering of $\mathcal{W}$ in $C[0, a]$, hence there is a finite open covering $\left\{\boldsymbol{B}_{\alpha_{i}}\right\}_{i=1}^{k}$. At last, $\left\{V_{\alpha_{i}}: \boldsymbol{B}_{\alpha_{i}} \subseteq V_{\alpha_{i}}\right\}_{i=1}^{k}$ is a covering of $\mathcal{W}$ and so $\mathcal{W}$ is compact in $C^{m}[-1,1]$.

Since $\overline{T(\mathcal{B})}^{C^{m}[-1,1]} \subseteq \overline{T(\mathcal{B})}^{C[0, a]}=\mathcal{W}, \overline{T(\mathbb{B})}$ is compact in $C^{m}[-1,1]$.

\section{Controllability}

Let $U$ be a Banach space. One will consider the following linear system:

$$
\begin{gathered}
x^{\prime}(t)=A x(t)+B u(t), \\
x(0)=x_{0}, \quad x_{0} \in D(A), \\
x(\theta)=\varphi(\theta), \quad \theta \in[-1,1],
\end{gathered}
$$

where $A: D(A) \subseteq N \rightarrow N$ is the infinitesimal generator of the semigroup $(S(t))_{t \geq 0}, B: U \rightarrow$ $N$ is a bonded linear operator, $u:[0, \infty) \rightarrow U$ is a strongly measurable, essentially bounded function.

In this section, we will study the controllability of the system (4.1). The mild solution of (4.1) is given by

$$
x(t)=S(t) x_{0}+\int_{0}^{t} S(t-s) B u(s) \mathrm{d} s
$$

Further, we will suppose that $\Omega$ is a separable weakly compact convex subset of $U$.

For $\tau>0$, the set

$$
\widetilde{\Omega}_{\tau}=\left\{u \in L_{U}^{\infty}[0, \tau]: u \in \Omega \text { a.e. }\right\}
$$


is called the set of admissible controls of (4.1), while the set

$$
A_{\tau}\left(x_{0}\right)=\left\{S(\tau) x_{0}+\int_{0}^{\tau} S(\tau-s) B u(s) \mathrm{d} s: u \in \tilde{\Omega}_{\tau}\right\}
$$

is called the set of accessible points of (4.1).

The system (4.1) is controllable if $0 \in A_{\tau}\left(x_{0}\right)$, for each $\tau>0$.

Let $x_{0}, \varphi, U, u, B$ be as (4.1). Consider for each $m \in \mathbb{N}$, the system

$$
\begin{gathered}
x^{\prime}(t)=A^{m} x(t)+B u(t), \\
x(0)=x_{0}, \quad x_{0} \in D(A), \\
x(\theta)=\varphi(\theta), \quad \theta \in[-1,1],
\end{gathered}
$$

where $B: U \rightarrow N_{m}$.

Note that, in this case, $B$ is also a bounded operator. In fact, since for each $u \in U$, $B u \in N_{m}$, we can write $B u=\left(B_{1} u, B_{2} u\right)$, where $B_{1}: U \rightarrow \mathbb{C}^{n}$ and $B_{2}: U \rightarrow M_{m}$ are bounded linear operators. If $\left\{u_{j}\right\}_{j=0}^{\infty} \subseteq U$ is a convergent sequence to $u$, then

$$
\begin{aligned}
\left\|B u_{j}-B u\right\|_{\mathbb{C}^{n} \times M_{m}} & =\left\|\left(\begin{array}{l}
B_{1} u_{j} \\
B_{2} u_{j}
\end{array}\right)-\left(\begin{array}{c}
B_{1} u \\
B_{2} u
\end{array}\right)\right\|_{\mathbb{C}^{n} \times M_{m}}=\left\|\left(\begin{array}{c}
B_{1} u_{j}-B_{1} u \\
B_{2} u_{j}-B_{2} u
\end{array}\right)\right\|_{\mathbb{C}^{n} \times M_{m}} \\
& =\left\|B_{1} u_{j}-B_{1} u\right\|_{\mathbb{C}^{n}}+\left\|B_{2} u_{j}-B_{2} u\right\|_{M_{m}} \leq\left\|B_{1} u_{j}-B_{1} u\right\|_{\mathbb{C}^{n}}+P_{m}\left(B_{2} u_{j}-B_{2} u\right)
\end{aligned}
$$

which converges to zero as $j$ tends to $+\infty$.

Let us consider $\Omega$ and $\widetilde{\Omega}_{\tau}$ as before.

The system (4.5) is controllable if $0 \in A_{\tau}^{m}\left(x_{0}\right)$, for each $\tau>0$, where

$$
A_{\tau}^{m}\left(x_{0}\right)=\left\{S^{m}(\tau) x_{0}+\int_{0}^{\tau} S^{m}(\tau-s) B u(s) \mathrm{d} s: u \in \tilde{\Omega}_{\tau}\right\}
$$

Lemma 4.1. $A_{\tau} x_{0}=\bigcap_{m=1}^{\infty} A_{\tau}^{m} x_{0}$.

Proof. If $z \in A_{\tau} x_{0}$, there exist $u \in \widetilde{\Omega}_{\tau}$ such that $z=S(\tau) x_{0}+\int_{0}^{\tau} S(\tau-s) B u(s) \mathrm{d}$. Since $S(\tau) x_{0}=$ $S^{m}(\tau) x_{0}$ and $S(\tau-s) B u(s)=S^{m}(\tau-s) B u(s)$, for all $m \in \mathbb{N}$, then $z \in \bigcap_{m=1}^{\infty} A_{\tau}^{m} x_{0}$. If $z \in$ $\bigcap_{m=1}^{\infty} A_{\tau}^{m} x_{0}$, then there exists, for every $m \in \mathbb{N}, u_{m} \in \widetilde{\Omega}_{\tau}$, such that $z=S^{m}(\tau) x_{0}+\int_{0}^{\tau} S^{m}(\tau-$ s) $B u_{m}(s) \mathrm{d} s$. In particular, for $m=1, z=S^{1}(\tau) x_{0}+\int_{0}^{\tau} S^{1}(\tau-s) B u_{1}(s) \mathrm{d} s$ and as $S(\tau) x_{0}=$ $S^{1}(\tau) x_{0}$ and $S(\tau-s) B u_{1}(s)=S^{1}(\tau-s) B u_{1}(s)$, then $z=S^{1}(\tau) x_{0}+\int_{0}^{\tau} S^{1}(\tau-s) B u_{1}(s) \mathrm{d} s$, that is, $z \in A_{\tau} x_{0}$. 
Before stating the main result on controllability, we need to prove the following lemma.

Lemma 4.2. The map $T_{t}: M_{m} \rightarrow M_{m}$, defined by $T_{t}(u)(\theta)=u(t+\theta)$, is compact.

Proof. To prove this lemma, we will follow the next five steps. compact

(1) The Kondrasov's Theorem [12,13] gives that the following canonical injection is

$$
H^{m}(-1,1) \stackrel{c}{\hookrightarrow} C^{0}([-1,1]) \quad \text { if } m \in \mathbb{N},
$$

where

$$
H^{m}(-1,1)=\left\{f \in L^{2}(-1,1): f^{(k)} \in L^{2}(-1,1), k=1, \ldots, m\right\}
$$

with $f^{(k)}$ being the derivative of order $k$ in the sense of distributions. $H^{m}(-1,1)$ is endowed with the norm

$$
\|f\|_{m}^{2}=\sum_{k=0}^{m} \int_{-1}^{1}\left|f^{(k)}(t)\right|^{2} \mathrm{~d} t
$$

This implies that $H^{m}[-1,1]$ is a Banach space.

(2) Using (1), for $m=1, H^{1}[-1,1] \stackrel{c}{\hookrightarrow} C^{0}([-1,1])$. Thus, for $m \geq 1$, if $f \in H^{m+1}(-1,1)$, then $f^{(m)} \in H^{1}(-1,1)$. Therefore, $H^{m+1}(-1,1) \stackrel{c}{\hookrightarrow} C^{m}([-1,1])$.

(3) By (2) and the definition of $M_{m+1}$, it follows that

$$
M_{m+1} \subseteq C^{m+1}([-1,1]) \subseteq H^{m+1}(-1,1) \stackrel{c}{\hookrightarrow} C^{m}([-1,1])
$$

Hence, for every bounded sequence $\left\{f_{k}\right\}_{k \in \mathbb{N}}$ in $M_{m+1}$ with the topology of $C^{m}([-1,1])$, it can be found a convergent subsequence $\left\{f_{k_{l}}\right\}_{l \in \mathbb{N}} \subseteq\left\{f_{k}\right\}_{k \in \mathbb{N}}$ that converges to $f$ in $C^{m}([-1,1])$; but $f^{(m)}$ is continuous and $f^{(m+1)} \in L^{2}(-1,1)$. Thus $\left\{f_{k_{l}}^{(m+1)}\right\}_{l \in \mathbb{N}}$ converges to $f^{(m+1)}$ a.e, and so this subsequence converges to $f$ in $M_{m+1}$.

(4) To prove that $T_{t}$ is compact, it must be proven that for every bounded sequence $\left\{u_{i}\right\}_{i \in \mathbb{N}}$ in $M_{m}$, a subsequence $\left\{u_{i_{j}}\right\}_{j \in \mathbb{N}}$ can be found such that the sequence $\left\{T_{t}\left(u_{i_{j}}\right)\right\}_{j \in \mathbb{N}}$ converges in $M_{m}$. As $T_{t}\left(u_{i}\right)(\theta)=u_{i}(t+\theta)$, then the previous fact is equivalent to obtain a convergent subsequence $\left\{u_{i_{j}}\right\}_{j \in \mathbb{N}}$ from $\left\{u_{i}\right\}_{i \in \mathbb{N}}$ in $M_{m}$.

(5) Using (3) and the conclusion of (4), it is obtained that for all $t \in \mathbb{R}, T_{t}$ is compact as a bounded linear operator from $M_{m}$ to $M_{m}$.

Theorem 4.3. $0 \in A_{\tau} x_{0}$ for each $\tau>0$ (i.e., the system (4.1) is controllable) if and only if

$$
x^{*}\left(S^{m}(\tau) x_{0}\right)+\int_{0}^{\tau} \max _{u \in \Omega} x^{*}\left(S^{m}(t) B u\right) \mathrm{d} t \geq 0
$$

for every $m \in \mathbb{N}$ and each $x^{*} \in\left(N_{m}\right)^{*}$. 
Proof. First, we will prove that $S^{m}(t)$, for all $t \geq 0$, is compact, for all $m \in \mathbb{N}$; in fact,

$$
S^{m}(t)\left(\begin{array}{c}
r \\
\varphi(\cdot)
\end{array}\right)=\left(\begin{array}{c}
x(t) \\
x(t+\theta)
\end{array}\right)=\left(\begin{array}{c}
x(t) \\
0
\end{array}\right)+\left(\begin{array}{c}
0 \\
x(t+\theta)
\end{array}\right)
$$

The first term has finite rank, therefore, it is compact. According to Lemma 4.2, the second term is compact in $M_{m}$. Consequently, $S^{m}(t)$ is compact, as an operator in $L\left(\mathbb{C}^{n} \times M_{m}\right)$. In particular, it is compact as an operator in $L\left(N_{m}\right)$. Hence, the adjoint $\left(S^{m}(t)\right)^{*}$ is strongly continuous in $(0,+\infty)$ (see, e.g., [14]). On the other hand, in view of Lemma 4.1, $0 \in A_{\tau} x_{0}$ if and only if $0 \in A_{\tau}^{m} x_{0}$, for all $m \in \mathbb{N}$ and this is true, after Theorem 1.1 of Bárcenas-Diestel [5], if and only if $x^{*}\left(S^{m}(\tau) x_{0}\right)+\int_{0}^{\tau} \max _{u \in \Omega} x^{*}\left(S^{m}(t) B u\right) \mathrm{d} t \geq 0$ for all $m \in \mathbb{N}$ and each $x^{*} \in\left(N_{m}\right)^{*}$.

\section{References}

[1] A. Rustichini, "Functional differential equations of mixed type: the linear autonomous case," Journal of Dynamics and Differential Equations, vol. 1, no. 2, pp. 121-143, 1989.

[2] A. Rustichini, "Hopf bifurcation for functional-differential equations of mixed type," Journal of Dynamics and Differential Equations, vol. 1, no. 2, pp. 145-177, 1989.

[3] K. A. Abell, C. E. Elmer, A. R. Humphries, and E. S. Van Vleck, "Computation of mixed type functional differential boundary value problems," SIAM Journal on Applied Dynamical Systems, vol. 4, no. 3, pp. 755-781, 2005.

[4] H. J. Hupkes, Invariant Manifolds and Applications for Functional Differential Equations of Mixed Type, Universiteit Leiden, Leiden, The Netherlands, 2007.

[5] D. Bárcenas and J. Diestel, "Constrained controllability in nonreflexive Banach spaces," Quaestiones Mathematicae, vol. 18, no. 1-3, pp. 185-198, 1995.

[6] V. Iakovleva and C. J. Vanegas, "On the solution of differential-difference equations with delayed and advanced arguments," in Proceedings of the Colloquium on Differential Equations and Applications (EJDE '05), vol. 13, pp. 57-63, Southwest Texas State University, San Marcos, Tex, USA, 2005.

[7] V. Iakovleva and C. J. Vanegas, "Spectral analysis of strongly continuous semigroups associated to solutions of differential-difference equations of mixed-type," In press.

[8] R. F. Curtain and H. Zwart, An Introduction to Infinite-Dimensional Linear Systems Theory, vol. 21 of Texts in Applied Mathematics, Springer, New York, NY, USA, 1995.

[9] R. C. James, "Weakly compact sets," Transactions of the American Mathematical Society, vol. 113, pp. 129-140, 1964.

[10] G. Peichl and W. Schappacher, "Constrained controllability in Banach spaces," SIAM Journal on Control and Optimization, vol. 24, no. 6, pp. 1261-1275, 1986.

[11] K. Yosida, Functional Analysis, vol. 123, Springer, New York, NY, USA, 4th edition, 1974, Die Grundlehren der Mathematischen Wissenschaften.

[12] R. A. Adams, Sobolev Spaces, vol. 65, Academic Press, San Diego, Calif, USA, 1975, Pure and Applied Mathematics.

[13] P. G. Ciarlet, The Finite Element Method for Elliptic Problems, vol. 4, North-Holland Publishing, Amsterdam, The Netherlands, 1978, Studies in Mathematics and Its Applications.

[14] D. Bárcenas and L. G. Mármol, "On the adjoint of a strongly continuous semigroup," Abstract and Applied Analysis, vol. 2008, 11 pages, 2008. 


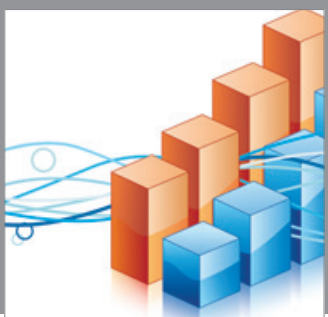

Advances in

Operations Research

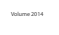

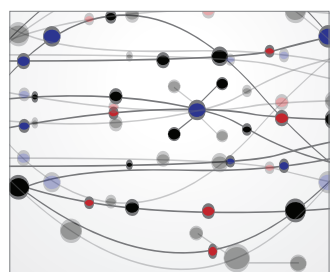

\section{The Scientific} World Journal
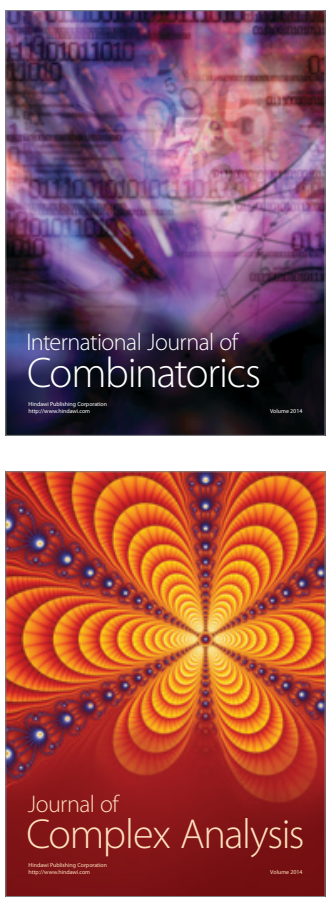

International Journal of

Mathematics and

Mathematical

Sciences
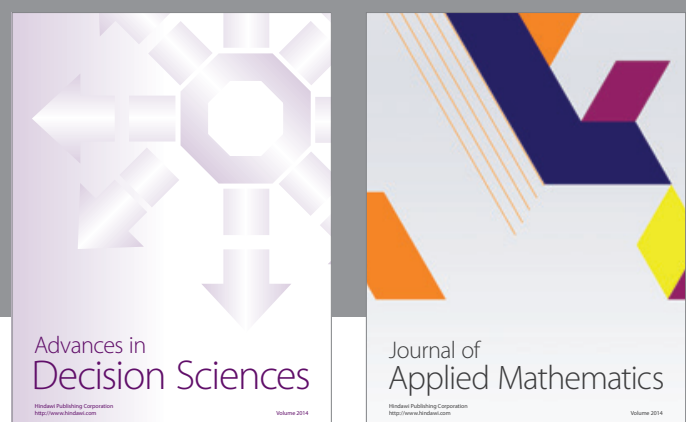

Journal of

Applied Mathematics
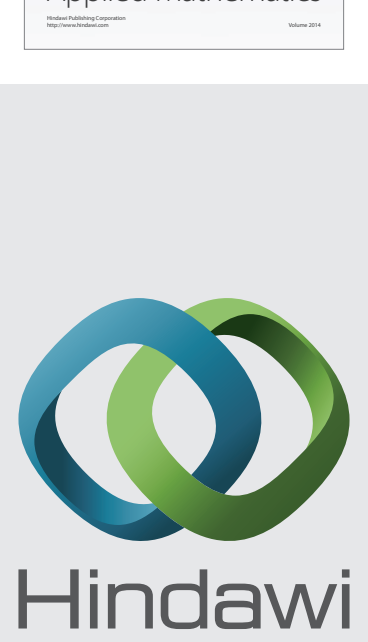

Submit your manuscripts at http://www.hindawi.com
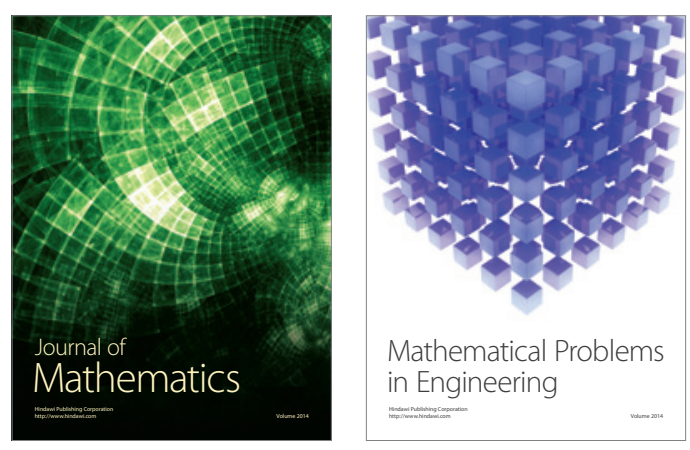

Mathematical Problems in Engineering
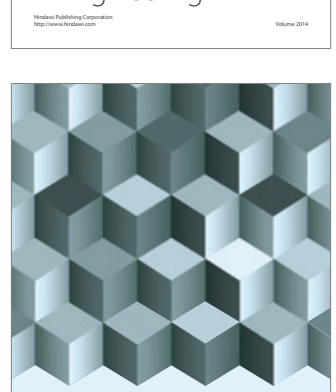

Journal of

Function Spaces
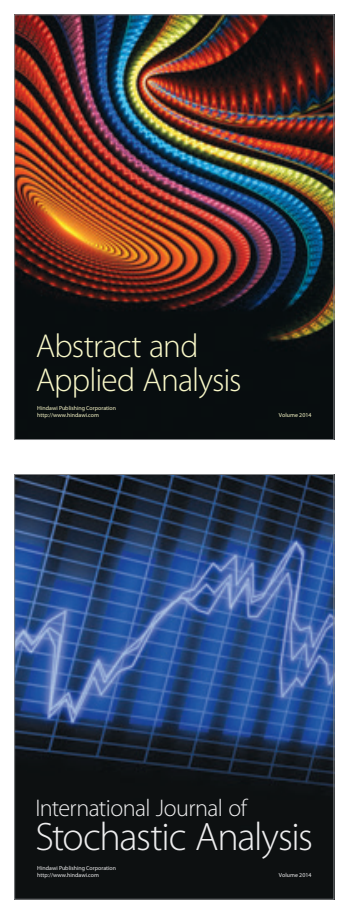

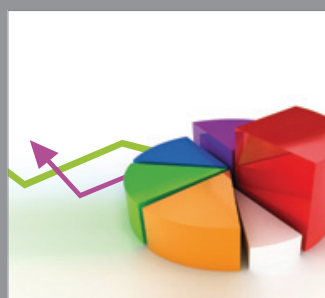

ournal of

Probability and Statistics

Promensencen
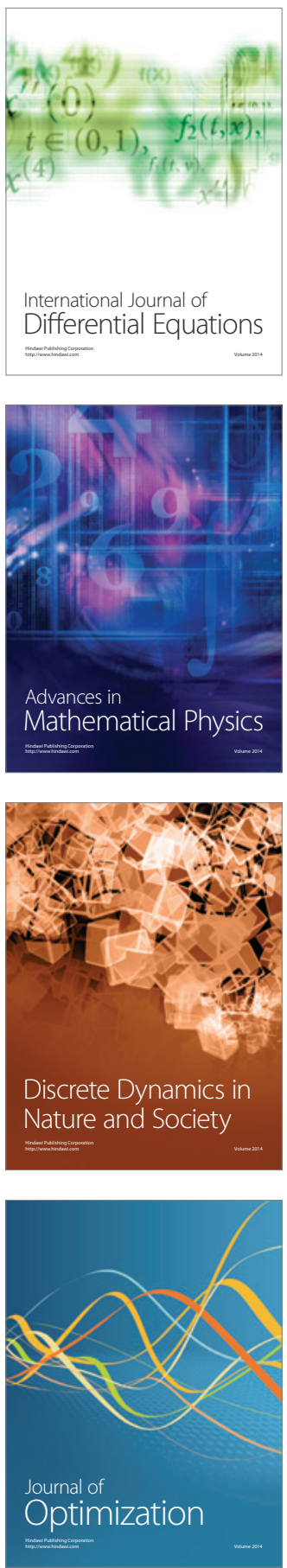